\title{
DÉVELOPPEMENT DES POUVOIRS DE L'ÉTAT FÉdÉRAL AMÉRICAIN DANS UN CONTEXTE DE CRISE ÉCONOMIQUE: UNE ÉTUDE DE CAS PORTANT SUR LE NEW DEAL ET LE SYSTÈME DES POIDS ET CONTREPOIDS
}

\author{
Par David Houle
}

\begin{abstract}
This article analyses the principles of the American system of 'checks and balances' during the period of economic and social turmoil under the presidency of Franklin D Roosevelt - creator of the New Deal policy. The author describes the fundamental principles of the American system of 'checks and balances,' while commenting on historical context and the American political system in general. Finally, the author concludes with the suggestion that the federal executive capitalized on the Great Depression by increasing its power over the United State's administration.
\end{abstract}

\section{Introduction}

Inscrit à l'intérieur de la Constitution américaine de 1787, le principe de « checks and balances », que nous traduirons en français par « poids et contrepoids ", fut inspiré de la crainte de l'arbitraire tel qu'il fut exercé par les détenteurs du pouvoir monarchique. " Car tel est mon bon plaisir », affirmait Louis XIV, monarque absolu de la France, pour justifier ses décisions. C'est en réaction de ce qu'elles percevaient comme étant les abus de ce système que les treize colonies décidèrent de rompre leurs liens avec la métropole britannique et de bâtir un ordre constitutionnel nouveau. Ils eurent alors l'idée, inspirés par des philosophes de l'époque, de diviser le pouvoir et 
de le remettre entre les mains de plusieurs institutions qui se feraient concurrence. Le principe des poids et contrepoids venait de trouver sa première application pratique. Dans un premier temps, leur attention se porta principalement sur les pouvoirs législatif et exécutif. Le pouvoir judiciaire allait se développer un peu plus tard dans un esprit que n'auraient pas renié les Pères de la confédération.

Cet article a comme principal objectif d'étudier le fonctionnement du principe des poids et contrepoids lors de la période de bouleversements économiques et sociaux initiés sous la présidence de Franklin D. Roosevelt, qui fut désigné comme étant le New Deal. Dans un premier temps, nous verrons les bases théoriques qui sous-tendent le principe des poids et des contrepoids. Par la suite, nous décrirons le contexte historique dans lequel s'inscrit la période qui fait l'objet de cet article. Finalement, nous étudierons le fonctionnement du principe des poids et contrepoids dans ce contexte et nous verrons comment le gouvernement fédéral américain, principalement l'exécutif, a tiré profit de cette situation pour accroître ses prérogatives.

\section{Bases théoriques du système politique américain}

Expérience novatrice à une époque où le pouvoir était essentiellement détenu par des monarques s'appuyant sur le droit divin, l'expérience constitutionnelle américaine allait marquer l'Histoire et influencer les révolutions à venir. Afin de créer leurs nouvelles institutions, les constituants de 1787 durent s'appuyer sur des bases philosophiques développées par certains de leurs contemporains. Dans notre étude concernant le système des poids et contrepoids, nous allons en retenir principalement deux : John Locke et Charles de Secondat, baron de La Brède et de Montesquieu.

Pour Locke, "[...] l'autorité du gouvernement dépend du consentement des administrés $[\ldots] »^{I}$. Les monarchies de droit divin seraient donc dépourvues de toute légitimité. De plus, "[...] le pouvoir législatif est le pouvoir suprême, les lois positives (par opposition au droit naturel) exprimant la volonté du peuple $\|^{2}$. C'est ce qui explique l'insistance des Pères de la confédération sur le pouvoir législatif et l'importance du Congrès des États-Unis qui fait l'objet du premier article de la Constitution américaine. Pour ce philosophe d'origine anglaise, l'individu et la propriété privée sont d'une importance primordiale. Pour les préserver et favoriser 
l'épanouissement personnel, il faut parvenir à limiter le pouvoir des gouvernants et les possibilités de décisions arbitraires qu'il peut engendrer. Deux principes fondamentaux, qui feront parties de l'expérience politique américaine, apporteront cette protection à la liberté individuelle : la séparation des pouvoirs ${ }^{3}$, qui se traduira aux États-Unis par la distinction entre le pouvoir exécutif, législatif et judiciaire, et le concept des « poids et contrepoids », qui s'exprimeront dans l'expérience américaine par les différentes compétences autonomes confiées par la Constitution aux trois pouvoirs. Pour sa part, Montesquieu a poursuivit les travaux de Locke en ce qui concerne la séparation des pouvoirs. Il affirme que " [dans] un gouvernement représentatif, pour que les représentants n'abusent pas $d u$ pouvoir, il est nécessaire de séparer et de distribuer le pouvoir public dans des organes distincts du gouvernement [...] $»^{4}$ et de faire en sorte que $4[\ldots]$ par la disposition des choses, le pouvoir arrête le pouvoir. ${ }^{5} \mathrm{Ce}$ philosophe croit que "[la] distinction tripartite des pouvoirs, assortie de leur association polémique, est la condition essentielle d'un gouvernement modéré. ${ }^{6}$ Par ailleurs, Montesquieu a reconnu l'existence du pouvoir judiciaire omis par Locke.

\section{L'expérience américaine}

Les constituants se sont réunis dans la ville de Philadelphie en 1787 pour mettre au point la Constitution d'un nouvel État, à la suite de l'indépendance américaine et de l'échec relatif, une fois la guerre contre la métropole passée, de l'union confédérale alors en vigueur. Suivant de nombreuses inspirations, dont celle de Locke et de Montesquieu, ils s'entendirent pour créer un État de type fédéral, caractérisé par la mise en place de trois pouvoirs autonomes et séparés, auxquels ils confièrent des pouvoirs limitant ceux exercés par les autres instances.

\section{Pouvoirs du législatif}

Le pouvoir législatif est lui-même réparti entre deux instances: le Sénat et la Chambre des représentants. Dans leur expression contemporaine (et pour la période qui nous intéresse soit de 1929 à 1939), les membres de ces deux chambres sont élus par la population pour des mandats de six ans, en ce qui concerne le Sénat, et de deux ans pour la Chambre des représentants. Encore aujourd'hui, il s'agit du plus puissant pouvoir législatif connu. De façon générale, il possède le pouvoir de légiférer, de proposer un amendement à la Constitution, de superviser et de contrôler les pouvoirs 
publics, de passer outre au veto présidentiel (avec un vote des $2 / 3$ dans chaque chambre) et d'impeachment à l'endroit du président et de l'ensemble des fonctionnaires fédéraux. De plus, le Sénat possède des pouvoirs spécifiques. C'est lui qui approuve les nominations du président et adopte les traités. Finalement, le Congrès décide du nombre de juges à la Cour suprême.

\section{Pouvoirs de l'exécutif}

Pour sa part, le pouvoir exécutif est moniste et par conséquent concentré entre les mains du président des États-Unis qui est à la fois chef du gouvernement et de l'État. Le président est élu de façon indirecte par les citoyens américains pour un mandat de quatre ans. En principe, le président ne peut poursuivre plus de deux mandats. Cependant, il s'agit d'une convention qui ne fut inscrite à la Constitution (par le vingt-deuxième amendement) qu'en 1951. Cela a permis au président Franklin D. Roosevelt de faire quatre mandats consécutifs. Le président possède les pouvoirs régaliens, notamment le droit de grâce, et il est le commandant en chef des forces armées. De plus, il est le représentant des États-Unis. À ce titre, il négocie les traités et nomme les ambassadeurs.

\section{Pouvoirs du judiciaire}

Dans notre étude de cas, nous restreindrons volontairement le pouvoir judiciaire, très complexe aux États-Unis, à la Cour suprême fédérale. Cette dernière est, et était entre 1929 et 1939, composée de neuf juges (le « chief justice » et huit « associate justice ») nommés à vie par le président avec l'accord du Sénat. La Cour peut annuler des décrets présidentiels et déclarer des lois inconstitutionnelles. Le contrôle de la constitutionnalité des lois n'était pas une prérogative que la Constitution attribuait à la Cour suprême. C'est seulement en 1803 que la Cour obtient ce pouvoir à la suite du jugement rendu dans la cause Marbury c. Madison. ${ }^{7}$ Elle statua alors qu'elle ne pouvait rendre une décision dans l'affaire, car le Judiciary act de 1789, qui l'habilitait à entendre la cause en première instance, était contraire à la Constitution. Ce jugement tira le juge en chef de l'époque, John Marshall, d'une situation délicate et créa un précédent primordial pour le système politique américain.

\section{Les États}


Si le pouvoir, au niveau fédéral, est limité et partagé entre différentes institutions, il est important de souligner qu'il est également partagé entre le gouvernement central et les États fédérés. La Constitution américaine prévoit des pouvoirs spécifiques à ces derniers et d'autres à l'État central. Par exemple, le commerce interétatique est régi par le gouvernement fédéral alors que les États doivent s'assurer de régir le commerce à l'intérieur de leurs frontières.

\section{Contexte historique : le New Deal}

Tout au long de l'histoire américaine, les pouvoirs dévolus par la Constitution à chaque institution furent interprétés différemment. Le contexte général de la société américaine, à un moment donné, conditionne les relations entre les différentes composantes gouvernementales. Afin de comprendre l'action des poids et contrepoids tel qu'elle s'exerce à l'occasion du New Deal, nous décrirons brièvement la situation qui se présentait lors de l'élection de Franklin D. Roosevelt à la présidence en 1933.

\section{La crise économique de 1929}

Les causes de la grande crise économique de 1929 nous semblent encore mystérieuses, bien que certains économistes et historiens aient développé des thèses pour tenter de l'expliquer. Pour Harold James, professeur d'histoire moderne à l'Université de Princeton, « [...] lorsque les gouvernements essayèrent de rétablir l'étalon-or [après la première guerre mondiale], ils s'aperçurent que les mouvements de capitaux à court terme était beaucoup plus instables [...] pour plusieurs raisons : les gravent problèmes structurels résultant du conflit, l'imbroglio politique autour de la dette de guerre et des réparations, et de profondes discordances entre les politiques des plus grands pays. $\|^{8}$. Ces facteurs ont contribués à engendrer une incertitude qui aurait conduit à la mise en place d'entraves au commerce international. Ces barrières auraient engendré une chute des exportations de nombreux pays, réduisant par conséquent la demande pour un grand nombre de produits et les prix.

La déflation et la baisse de la demande entraînèrent la paupérisation des agriculteurs, incapables de vendre leur production, ainsi que la faillite de 
nombreuses industries. Une autre conséquence du climat d'incertitude fut l'éclatement de la bulle spéculative qui causa le krach boursier d'octobre 1929. C'est alors que s'enclencha un processus de destruction de la masse monétaire par, dans un premier temps, l'effondrement des valeurs en bourse et, dans un deuxième temps, le vent de panique qui mena des centaines d'américains, dans un geste de désespoir, à retirer leurs économies des banques qui furent ainsi acculées à la faillite.

Les dépôts n'étant pas assurés, beaucoup d'américains ont alors tout perdu, ce qui diminua encore la quantité de monnaie dans l'économie. Pour couronner le tout, la fermeture d'un grand nombre de banques entraîna le ralentissement du processus de création monétaire et le système de l'étalonor contraignit le gouvernement à ne pas faire usage de la presse à billets (puisqu'il devait respecter une parité fixe avec l'or).

C'est ce qui amène André Kaspi à affirmer que «[...] les récoltes de 1930 et de 1931 sont abondantes et donc invendables. Dans les villes, on meurt de faim. $\gg{ }^{9}$ Personne n'a d'argent pour acheter les productions agricole et industrielle, la demande agrégée s'effondre entraînant avec elle le Produit National Brut (PNB), qui passe de 104,4 milliards en 1929 à 72,7 milliards de dollars en 1933 (en dollars constant de 1929) ${ }^{10}$.

Par ailleurs, le chômage atteint des sommets et les prix chutent. Le président Herbert Hoover, en poste depuis 1928, " [...] a essayé divers remèdes: les déclarations rassurantes [...], une politique volontariste pour encourager banquiers, syndicats et hommes d'affaires à embaucher ou du moins à maintenir le niveau des salaires, des mesures douanières [qui auraient empiré la crise selon l'analyse du professeur James], la mise sur pied de la Reconstruction Finance Corporation ayant pour mission de distribuer des fonds aux entreprises en difficulté.» ${ }^{11}$ Cependant, Hoover répugne à avoir recours à l'interventionnisme étatique, imprégné qu'il est de l'image du bon gestionnaire qui maintient l'équilibre budgétaire à tout prix. La crise peut alors continuer à s'empirer...

\section{Les élections présidentielles de 1932}

C'est dans ce contexte que les américains sont appelés aux urnes le 8 novembre 1932. Le candidat du parti démocrate Franklin D. Roosevelt obtient 22809638 voix (57,42\% des suffrages exprimés) contre 17758901 pour le président Hoover. Au succès démocrate à l'élection présidentielle 
correspond également un succès aux élections législatives. Les démocrates possèdent une majorité dans les deux chambres puisqu'ils détiennent 310 représentants sur 435 et 60 sénateurs sur une possibilité de $96^{12}$.

Dans son discours de la victoire, le 4 mars 1933, le président déclare que « [...] les marchands du Temple [référence aux spéculateurs et autres businessmans principaux auteurs de la crise selon Roosevelt] ont abandonné leurs sièges dans le temple de la civilisation. Nous pouvons à présent restaurer les antiques vérités dans ce temple. La restauration suivra l'application des valeurs sociales, plus nobles que le seul profit matériel. ${ }^{13}$ Le coup d'envoi d'une multitude de mesures interventionnistes est lancé, l'aventure du New Deal vient alors de commencer et ne s'arrêtera que lorsque les États-Unis auront été durablement transformés par le gouvernement fédéral.

\section{Analyse de l'utilisation du pouvoir par les acteurs dans ce contexte: le principe du « poids et contrepoids $॥$ en action}

Dans le même discours le président Roosevelt déclare « [qu'il] faut espérer que l'équilibre normal entre le pouvoir exécutif et le pouvoir législatif sera totalement adéquat pour que nous réalisions la tâche sans précédente qui nous attend.». Il ira même jusqu'à affirmer que "[suivant] mes devoirs constitutionnels, je suis prêt à recommander les mesures que peut réclamer une nation malade dans un monde malade [...] Je demanderai au Congrès de me donner l'instrument pour affronter la crise, c'est-à-dire des pouvoirs aussi étendus que si nous étions envahi par un ennemi étranger. $»^{14}$

\section{Les Cent Jours (mars-juin 1933): collaboration entre le président et le Congrès}

Dans un premier temps, l'équilibre des pouvoirs fut à la faveur de la présidence. Le Congrès lui accorda les pouvoirs exceptionnels qu'il demandait. Cela déclancha une période de cent jours d'intenses travaux législatifs et de collaboration entre les pouvoirs législatif et exécutif. À ce 
moment, rien ne semblait pouvoir freiner l'exercice du pouvoir présidentiel qui pouvait compter sur une majorité sympathique à sa cause dans les deux chambres. Pourtant, aux États-Unis, la ligne de parti n'existe que le temps des élections. Par conséquent, le fait que le président et les majorités dans les deux chambres soient du même parti ne garanti rien.

Pour expliquer la situation qui prévalait de mars à juin 1933, Alain Kaspi recourt à plusieurs explications ${ }^{15}$. Premièrement, à la Chambre des représentants, 131 membres sont des nouveaux élus démocrates sans expérience parlementaire et qui ont profité de la popularité de Roosevelt pour se faire élire. Ils savent que pour conserver leur siège la situation économique doit s'améliorer. Ils sont donc prêts à suivre Roosevelt. Deuxièmement, le président peut compter sur l'appui des leaders démocrates dans les deux chambres. Troisièmement, les républicains sont totalement déroutés par la défaite cinglante qu'ils viennent de subir et finalement, le président se comporte en législateur en chef car, bien qu'il n'ait pas le pouvoir de proposer des lois, il délimite le cadre du débat politique, suggère des échéances et des choix. Le président va également utiliser " [...] les armes traditionnelles de la présidence : invitation à la Maison Blanche, la photographie de l'invité en compagnie du président pour le journal local, la promesse de quelques prébendes. $»^{16}$

Finalement, le président Roosevelt, pour la première fois de l'histoire américaine, s'appuiera sur une équipe de politologues, de juristes et d'économistes: le Brain Trust. La plupart sont d'éminents professeurs universitaires. Ils seront, ainsi que leurs étudiants les plus brillants, à l'origine de plusieurs des projets de loi proposés au Congrès. Par leur présence au côté du président, ils renforcent sa crédibilité et son influence. Cette dernière s'étend également en raison de des talents de communicateur de Roosevelt et de la sympathie qu'il sait créer chez ses interlocuteurs.

À la fin de la période des Cent Jours la plupart des projets de loi présidentiels furent adoptés. Les banques, fermées sous la période d'Hoover, furent autorisées à rouvrir et une série de lois (Agriculture Adjustement Act (AAA), National Industrial Recovery Act (NIRA), etc.) furent adoptées.

Une multitude d'organismes publics ont également été créée pour réguler une partie de l'économie ou donner du travail aux chômeurs (National Recovery Board (NRA), Tennesse Valley Autority (TVA), Public Works Administration (PWA), etc.). Par ailleurs, soulignons que le président Roosevelt, pouvant compter sur une grande majorité des membres du 
Congrès, n'hésita pas à utiliser son droit de veto dans la mesure où il savait que ses adversaires ne pourraient réunir une majorité suffisante pour le renverser. Il le fit 635 fois, ce qui demeure un record inégalé ${ }^{17}$.

\section{Création de l'État-providence (1934-36) : le réveil de la Cour Suprême}

La Cour suprême fédérale fut la première à actionner les mécanismes des poids et des contrepoids avec succès. Même si les démocrates obtiennent encore des gains aux élections de mi-mandat tenues en 1934 les membres du Congrès " [...] ne veulent plus entendre parler de réforme puisque leurs électeurs ne s'enthousiasme plus pour les transformations sociales. En outre le président a distribué des places, donc son pouvoir de patronage $s$ 'épuise. ${ }^{18}$. De plus, un doute plane sur la réélection de Roosevelt aux élections présidentielles de 1936. Tous ces facteurs concourent à affaiblir son influence sur le Congrès.

C'est dans ce contexte que la Cour suprême rend un arrêt unanime au sujet du NIRA. Selon la Cour, cette loi irait à l'encontre de deux principes constitutionnelles : le fédéralisme, car la loi fait intervenir le gouvernement central dans le commerce à l'intérieur des États, et la séparation des pouvoirs, puisque seul le pouvoir législatif peut faire les lois (et, selon la Cour, l'exécutif est intervenu dans le processus). Cependant, le NIRA arrivait à échéance le mois suivant puisqu'il comportait une clause crépusculaire. Tout de même, le message que veulent envoyer les juges au président semble clair : il faut mettre fin aux centralisations, la Cour ne laissera plus la présidence agir dans cette direction ${ }^{19}$. Roosevelt dénonce alors l'interprétation étroite de la Cour suprême et "[il] se servira de l'émoi causé par la Cour pour préparer une nouvelle législation [...] à la fin d'août 1935, l'essentiel du Welfare State sera mis en place.» Cette nouvelle conception du rôle de l'État repose sur trois piliers : la sécurité sociale, l'aide aux chômeurs et la justice fiscale.

\section{La fin du New Deal (1937-39); renforcement du pouvoir exécutif federal}

Les sceptiques furent confondues et Roosevelt fut réélu pour son deuxième mandat le 3 novembre 1936 par une large majorité d'américains. En fait, il obtient 5 millions de voix de plus qu'en 1932. Le message semble 
là aussi très clair. Les américains, dont un grand nombre furent directement aidés par les interventions du gouvernement fédéral, semblent vouloir poursuivre les réformes. Cependant, Roosevelt s'inquiète du sort que pourrait réserver une Cour suprême conservatrice à ces nouvelles mesures législatives ainsi qu'à celles qui ont déjà été adoptées. En fait, " [...] de 1920 à 1933, elle $s$ 'était [la Cour suprême] prononcée vingt-deux fois contre des lois adoptées par le Congrès fédéral. ${ }^{20}$ C'est alors que va se jouer un épique bras de fer entre la présidence et la Cour.

\section{L’affaire de la Cour Suprême}

Le 5 février 1936 le président réunit à la Maison Blanche les principaux leaders du Congrès et les membres de son cabinet. À cette occasion, il leur fait part d'un message qu'il entend envoyer aux deux chambres. Le président demande "[qu'à] chaque fois que l'un des juges atteint l'âge de soixante-dix ans et ne part pas à la retraite, un nouveau juge soit nommé. Aux membres de la Cour s'ajouteraient 6 juges au maximum. $»^{21}$.

Les membres du Congrès présents sont mis devant le fait accompli. Il est dix heures et le texte sera présenté à midi au Congrès. Une copie a d'ailleurs déjà été distribuée aux journalistes. Le projet soulève un tollé de protestations. Pourtant, par le passé, la Cour avait déjà connu des modifications du nombre de ces membres. C'est alors que dans la tourmente du débat public engendrée par la proposition, qui ne fut pas acceptée, la Cour suprême céda aux pressions présidentielles. Un commentateur de l'époque affirma alors qu'il s'agissait d'un "[...] switch in time that saved nine. ${ }^{22}$

Par la suite, la Cour prit une série de décisions en faveur des mesures législatives du New Deal. Ce fut d'abord, le 29 mars 1936, une loi de l'État de Washington sur le salaire minimum qui fut déclarée constitutionnelle (alors qu'une loi semblable de l'État de New York avait été, quelques mois plus tôt, rejetée par la Cour). Par la suite, ce fut la loi Wagner, concernant les syndicats, et la loi sur la sécurité sociale. «[Peu] de temps après, Roosevelt eut l'occasion de nommer à la Cour suprême un candidat de son choix après le départ à la retraire du juge associé William Van Devanter [...] entre 1937 et 1943, il [nomma] huit autres juges à la Cour suprême [...] ${ }^{23}$. Ces nominations changèrent l'orientation idéologique de la Cour. 


\section{L'indépendance des membres du Congrès}

Bien que le président sembla l'emporter contre la Cour suprême, l'affaire contribua à renverser la coalition rooseveltienne au Congrès et, par conséquent, à stopper les réformes. Une légère embellie économique donna de l'eau au moulin des conservateurs qui affirmèrent que, la crise étant passée, rien ne justifiait de maintenir les « mesures exceptionnelles » du New Deal. Le président Roosevelt arriva également à ces conclusions. Le crédit fut resserré par les banques fédérales et les États-Unis ne tardèrent pas à connaître une deuxième récession qui les frappa en 1937. C'est alors que la présidence entame un virage et déclenche une autre série de mesures interventionnistes (aide au logement, réglementation des salaires et de la durée du travail, etc.).

Cependant, cette dernière période de turbulences économiques fit encore perdre à Roosevelt des appuis au Congrès. Certains démocrates n'hésitèrent plus à le critiquer. Un deuxième bras de fer se dessine. Ce sera cette fois entre le président et certains membres démocrates du Congrès. Roosevelt ciblera alors quelques uns d'entre eux et tentera d'empêcher leur réélection afin d'affermir sa poigne sur la majorité démocrates des deux chambres. Il n'hésitera pas à se déplacer pour appuyer les candidats qui lui sont favorables et échouera lamentablement puisque la grande majorité de ces adversaires seront réélus et, certains l'affirmeront, en raison même de l'hostilité présidentielle qui fut perçue négativement par l'électorat.

L'indépendance des membres du Congrès sembla alors préservée de l'influence trop directe du président. Cela contribua à renforcer le principe des poids et contrepoids. Le président ne peut pas nécessairement faire élire ceux qu'ils désirent et les membres du Congrès auront toujours à l'esprit les intérêts de leurs commettants lorsqu'ils voteront sur un projet de loi et non la volonté présidentielle. Cela est sans doute venu tempérer les craintes de ceux qui voyaient d'un mauvais œil le président américain imposer ses volontés à une époque marquée par l'émergence des dictatures dans le monde occidental.

\section{Conclusion}


À la lecture des différents documents qui furent les sources indispensables de cet article, nous sommes arrivés à la conclusion que le principe des poids et contrepoids entraîne les États-Unis, à de nombreux moments de leur histoire, dans l'immobilisme. Le processus législatif américain recèle d'innombrables obstacles allant de l'étude du projet de loi devant un président de commission, qui possède le pouvoir d'en déférer indéfiniment l'adoption, au vote d'un texte identique dans les deux chambres ou, le cas échéant, à la recherche d'un compromis dans un comité de conciliation. Sans parler du droit de veto multiforme du président et du droit de regard de la Cour suprême. Les Pères de la confédération américaine, comme la plupart des assemblées bourgeoises de leur époque, avaient comme principal souci de se protéger de l'arbitraire royal qui entravait la bonne conduite de leurs affaires. Le bonheur était quelque chose d'individuel. Pourvu que l'État cesse d'intervenir de façon intempestive dans la vie privée des gens et tout irait pour le mieux.

Les crises économiques et l'élargissement du droit de vote aux classes les plus pauvres de la société allaient changer les choses. L'égalité des droits et la liberté inscrites dans les constitutions occidentales ne voulaient pas dire grand-chose pour la grande majorité des gens condamnés à une existence misérable sans cesse bouleversée par des chocs extérieurs et dont l'exercice de la liberté consistait, durant les crises économiques, à parcourir les routes à la recherche d'un moyen de survivre. De temps à autre, des présidents énergiques, comptant sur le soutien de cet électorat désespéré, sur des réformateurs soucieux de préserver le système capitaliste de ses contradictions les plus évidentes et sur des évènements dramatiques, arrivèrent à mettre en action l'État fédéral américain.

Cependant, il n'est pas surprenant de constater que des réformes, jugées essentielles par un grand nombre de citoyens et d'intellectuels, n'arrivent toujours pas à voir le jour dans ce pays. C'est notamment le cas de l'assurance médicale généralisée, très vite oubliée par Roosevelt car touchant à de puissants intérêts particuliers et que Clinton, sans grand succès, tenta de réaliser. Le New Deal fut tout de même une période d'expansion du pouvoir fédéral en général, et du président en particulier. Ce dernier pu s'appuyer sur une technocratie naissante et créa un secteur public (notamment avec la création de la Tenesse Valley Autority). Fait totalement nouveau et sans précédent aux États-Unis, l'État fédéral allait à partir de ce moment jouer un rôle actif dans l'économie. D'autres présidents américains arriveront-ils à mettre sur pied des réformes d'envergures dans une société qui est caractérisée par une disparité grandissante entre riches et pauvres? La question vaut la peine d'être débattue. 


\section{ENDNOTES}

Orban 31.

${ }^{2}$ Ibid. 31.

${ }^{3}$ Notons que pour Locke les trois pouvoirs sont: le pouvoir exécutif, le pouvoir législatif et le pouvoir fédératif (au sens des relations entre une république, des individus et des États étrangers).

${ }^{4}$ Mercier et Duhamel 51.

${ }^{5}$ Ibid. 51.

${ }^{6}$ Duquette et Lamoureux 324.

${ }^{7}$ Orabn 293-294.

${ }^{8}$ Harold 2.

${ }^{9}$ Kaspi 207.

${ }^{10}$ Ibid. 15.

${ }^{11}$ Ibid. 175.

12 Ibid. 221-222.

${ }^{13}$ Ibid. 29.

${ }^{14}$ Ibid. 31.

${ }^{15}$ Ibid. 242-243.

${ }^{16}$ Ibid. 244.

${ }^{17}$ Orban 241.

${ }^{18}$ Kaspi 276.

${ }^{19}$ Ibid. 277.

${ }^{20}$ Ibid. 310. 
${ }^{21}$ Ibid. 308.

${ }^{22}$ Orban 296.

${ }^{23}$ Ibid. 296.

\section{Bibliographie}

Duquette, Michel et Diane Lamoureux. Les idées politiques: de Platon à Marx. Montréal: Presses de l'Université de Montréal, 1993.

Harold, James. "La libéralisation pourrait-elle faire marche arrière?" Finances \& Développement (décembre 1999): 11-14.

Kaspi, André. Franklin D. Roosevelt. Paris: Éditions Fayard, 1988.

Mercier, Benoit et André Duhamel. La démocratie: ses fondements, son histoire et ses pratiques. Québec: le Directeur Général des Élections du Québec, 2000.

Orban, Edmond. Le système politique américain: nouvelle édition. Montréal: Presses de l'Université de Montréal, 2001. 